\title{
Simulation of high-efficiency gyrotron with the system of multistage energy recovery
}

\author{
Pavel Trofimov ${ }^{1, *}$ and Oleg Louksha ${ }^{1}$ \\ ${ }^{1}$ Peter the Great St. Petersburg Polytechnic University, 195251, Polytechnicheskaya 29, \\ St. Petersburg, Russia
}

\begin{abstract}
The results of simulations of helical electron beam formation and collecting, as well as high frequency wave-particle interaction processes, in the moderate-power experimental gyrotron with the frequency of $74.2 \mathrm{GHz}$ are presented. Various methods of beam quality and electron efficiency improvement via optimization of electric and magnetic field distributions in the cathode region were realized. In the optimal operating regime with high pitch ratio and low velocity spread, the electron efficiency of about $46 \%$ was calculated for the gyrotron with the magnetron injection gun including a control electrode and a cathode with sectioned emission. In the gyrotron collector region, a system of 4-stage electron energy recovery was used for enhancement of total efficiency of the device. By improving the quality of the electron beam and efficient energy recovery in the collector region, the total efficiency of the gyrotron equal to $71.8 \%$ was achieved.
\end{abstract}

\section{Introduction}

Presently, gyrotrons hold leading positions among microwave radiation sources in the millimeter and submillimeter wavelength ranges in terms of output electromagnetic power. Powerful gyrotrons provide megawatts of power in long-pulse and quasi-continuous regimes for applications in heating and current control of fusion plasma. These devices are also used in high-resolution spectroscopy, material processing, diagnostics and other applications

The output power of a gyrotron depends on the efficiency of interaction of the helical electron beam (HEB) with the high-frequency field in the open resonator. Usually, the electronic efficiency of powerful gyrotrons does not exceed 30-35 \% [1-3]. Increasing electronic efficiency can be achieved by optimization of electron-optical system resulting in formation of the electron beam of high quality with large pitch ratio, low velocity and energy spreads and needed spatial structure [4]. Pitch ratio $\left(\alpha=v_{\perp} / v_{\|}\right)$determines the part of electron energy accumulated in transverse motion from which the power of electromagnetic wave in gyro-devices is extracted. The examples of such an optimization are megawatt level power gyrotrons manufactured by IAP RAS that characterize by the frequencies of 110 and $140 \mathrm{GHz}$ and the electronic efficiency of more than $40 \%,[2,5]$. Experimental studies aimed at HEB quality improvement were also carried out at SPbPU

\footnotetext{
*Corresponding author: trofpa@yandex.ru
} 
using a pulsed gyrotron with the operating frequency of $74.2 \mathrm{GHz}$ and the output power of about $100 \mathrm{~kW}$ [6-11]. As a result of the experiments, the electron efficiency of the gyrotron was increased up to $46 \%$ via optimization of electric and magnetic field distributions in the HEB formation area [11].

The total efficiency of a gyrotron can be enhanced by means of electron energy recovery systems. In such systems, the part of the residual electron beam energy that remained after the interaction with high-frequency electromagnetic wave in the resonator can be returned back to the electric circuit. The modern high-power gyrotrons are equipped with single-stage depressed collectors in which the deceleration of electrons occurs in the region between the body of the device and the isolated collector. Such systems can increase the total efficiency of the gyrotron to $50-55 \%[1,2,12]$. It should be noted that the thermal loading on the collector is also reduced through deceleration of electrons, which is crucial for powerful long-pulse and quasi-continuous gyrotrons. A further increase of total efficiency of gyrotrons can be obtained using multistage energy recovery systems in which separation of the beam fractions with different energy of electrons results in deposition of these fractions on the electrodes under different potentials (for example, [13]). As we know, multistage depressed collectors have not yet been implemented in gyro-devices, possibly due to specific features of propagation of electron beam with position and velocity spreads of electrons in the presence of moderately strong magnetic field in the collector region of these devices. New possibilities for separation of electrons are opened by using the method based on their drift in the crossed electric and magnetic fields [14-16]. At SPbPU, this method was used for development of a multistage energy recovery system in which electrons are separated in azimuthal magnetic and axial electric fields [17].

The paper presents the results of the combined simulation of the SPbPU gyrotron, which includes formation of HEB in the electron-optical system, interaction of electron beam with high-frequency field in the resonator, and propagation of spent electrons in the collector region. The aim of the study was to achieve the highest possible value of gyrotron total efficiency via increase of beam quality and implementation of multistage system of electron energy recovery. The simulation was performed using the CST Studio Suite software package [18]. The data obtained at different stages of the simulation were compared with the experimental results.

\section{Trajectory analysis of HEB in the gyrotron electron-optical system}

At the first stage of the simulation, trajectories of electrons in the electron-optical system between the cathode and the resonator were calculated. The formation of electron beam in this gyrotron is carried out by a triode-type magnetron injection gun (Fig. 1). The cathode block includes a control electrode which is used for changing the electric field distribution in the near-cathode region. Optimization of this distribution can be resulted in reduction of velocity spread and, as a result, in increase of beam average pitch ratio $[9,11]$. The values of main parameters of the SPbPU gyrotron are shown in Table 1. These values correspond to the designed operating regime of the gyrotron with an average pitch ratio of 1.3. In the calculations described below, parameters $U_{0}, I_{\mathrm{b}}, B_{0}, B_{\mathrm{c}}$ were changed to adjust the characteristics of the beam at the entrance of the resonator and hence to optimize the generated microwave power.

Due to the specific features of the collector with multistage recovery system, two regimes of thermionic emission were calculated. In the first regime, the cathode emits electrons uniformly from the whole emission strip. The beam consisted of 3600 electronic trajectories in this regime. In the second regime (2200 trajectories), there was no emission from two azimuthal emission strip sectors of 70 degrees each. The spread of initial thermal 


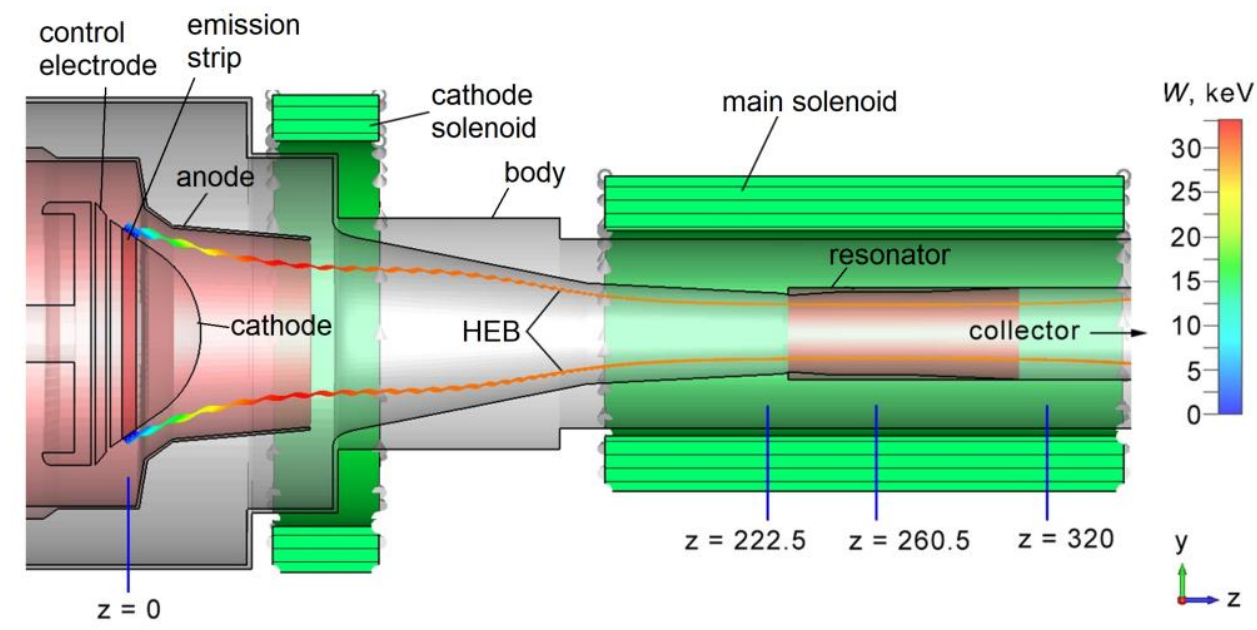

Fig. 1. Schematic drawing of the gyrotron model. Trajectories of electrons with different energies $W$.

velocities was taken into account in this simulation. In both regimes, the total electron beam current was $10 \mathrm{~A}$. The distribution of magnetic field was calculated by using parameters of the gyrotron coils and after that was imported to the trajectory analysis model. This model was divided into about 80 million mesh cells.

By varying the magnetic compression ratio $B_{0} / B_{\mathrm{c}}$, it is possible to adjust the HEB average radius in the resonator and consequently the output microwave power. In these calculations, as well as in earlier experiments [6], the value of $B_{0} / B_{\mathrm{c}}$ was changed by varying the number of turns of the cathode coil. The most efficient regime of the gyrotron was obtained at $B_{0} / B_{\mathrm{c}}$ $=17.01$, which corresponds to 26 turns of the cathode coil. At this value of $B_{0} / B_{\mathrm{c}}$, the voltages of anode $U_{\mathrm{a}}$ and control electrode $U_{\text {cont }}$ were determined to provide the operating regime of the gyrotron with low transverse velocity spread $\delta v_{\perp}$ and average pitch ratio $\bar{\alpha}$ in the range of $1.5-1.6$. Here the values of the HEB parameters refer to the central plane of the resonator $z=260.5 \mathrm{~mm}$ (Fig. 1). It should be noted that the maximum electronic efficiency of the gyrotron was experimentally measured in the regime with the value of $\bar{\alpha}$ equal to about 1.6 [11].

As a result of the trajectory analysis, the electron beam with $\bar{\alpha}=1.57, \delta v_{\perp}=2.8 \%$ (uniform cathode) and $\bar{\alpha}=1.52, \delta v_{\perp}=3.4 \%$ (sectioned cathode) was obtained at

Table 1. Parameters of experimental gyrotron.

\begin{tabular}{|l|l|}
\hline \multicolumn{1}{|c|}{ Parameter } & \multicolumn{1}{c|}{ Value } \\
\hline Accelerating voltage & $U_{0}=30 \mathrm{kV}$ \\
\hline Beam current & $I_{\mathrm{b}}=10 \mathrm{~A}$ \\
\hline Resonator magnetic field & $B_{0}=2.75 \mathrm{~T}$ \\
\hline Cathode magnetic field & $B_{\mathrm{c}}=0.152 \mathrm{~T}$ \\
\hline Operating mode & $\mathrm{TE}_{12,3}$ \\
\hline Operating frequency & $f_{0}=74.2 \mathrm{GHz}$ \\
\hline Resonator radius & $R_{0}=14.45 \mathrm{~mm}$ \\
\hline Emission strip average radius & $R_{\mathrm{c}}=35 \mathrm{~mm}$ \\
\hline
\end{tabular}


$B_{0} / B_{\mathrm{c}}=17.01, U_{\mathrm{a}}=8.4 \mathrm{kV}$ and $U_{\text {cont }}=-44.5 \mathrm{kV}$ (cathode voltage $U_{\mathrm{c}}=-30 \mathrm{kV}$, body voltage $U_{\text {body }}=0$ ). The values of $\delta v_{\perp}$ were defined as the standard deviation from the average value of transverse velocity. The sectioned cathode, as compared with the uniform cathode, is characterized by increased velocity and energy spreads as well as an additional radial displacement of electrons due to the action of crossed azimuthal electric and longitudinal magnetic fields [19]. The strong magnetic field provides conservation of the required electron distribution along the azimuthal coordinate for the sectioned cathode during propagation of the beam from the cathode to the collector entrance.

It should be noted that some factors, resulting in an additional velocity spread, have not been taken into account in this simulation: roughness of the emitting surface of the cathode strip, imperfections in manufacturing and alignment of tube elements, high-frequency fields associated with development of parasitic instabilities (see, for example, [4]). Highfrequency fields, in addition to increasing the velocity spread, can also cause a noticeable increase in the energy spread of electrons. Cathode surface roughness is the most important factor among listed in the case of well-aligned and adjusted electron-optical system in which the parasitic instabilities are suppressed. Typical roughness of emission surface with microstructure size of a few microns adds a few percent to the velocity spread [4, 20, 21].

The increased velocity spreads limits the possibilities of gyrotron operations at high average pitch ratio because realization of electron beam with high $\bar{\alpha}$ and $\delta v_{\perp}$ can lead to high percentage of electron reflection from the magnetic mirror resulting in development of the parasitic low-frequency oscillations in the trap between the cathode and the resonator $[4,6,22,23]$. Based on the experimental data obtained in the SPbPU gyrotron, the threshold coefficient of electron reflection from the mirror $\mathrm{R}_{\mathrm{th}}$ corresponding to excitation of these oscillations was determined to be approximately $1.7 \cdot 10^{-3}$ [10]. In the case of a Gaussian velocity distribution, this value of $\mathrm{R}_{\text {th }}$ is reached for the average pitch ratio $\bar{\alpha}=$ 1.57 at $\delta v_{\perp}=6.3 \%$, and for $\bar{\alpha}=1.52$ at $\delta v_{\perp}=6.7 \%$. In the experiments, the maximum electronic efficiency of the gyrotron $\eta_{\mathrm{el}}=46 \%$ was obtained in the regime with a calculated pitch ratio of $\sim 1.6$ and a small velocity spread, when parasitic low frequency oscillations were not recorded [11]. This gives grounds to assert that the regime with pitch ratio $\bar{\alpha}=1.5-1.6$, studied in this paper, can also be realized in the absence of electron reflection from the magnetic mirror.

The parameters of the HEB obtained from the trajectory analysis were used at the next stage of the simulation in which the interaction of the formed high quality HEB with the electromagnetic wave in the resonator was studied. The data were exported with help of special particle monitor available in CST Particle Studio placed in the cross-section plane with axial coordinate $z=222.5 \mathrm{~mm}$ (Fig. 1).

\section{Simulation of processes in the gyrotron resonator}

The resonator with the radius of $14.45 \mathrm{~mm}$ and the regular part length of $28 \mathrm{~mm}$ (Fig. 1) was designed for the working mode $\mathrm{TE}_{12,3}$. Preliminary calculations allowed to determine eigenmodes of the resonator and optimal average radius of the HEB. The particle-wave interaction was simulated using

the CST particle-in-cell solver. In this case, the model area was limited to input plane of the beam at $z=222.5 \mathrm{~mm}$ and the plane of the output port at $z=320 \mathrm{~mm}$ (Fig. 1). The calculation domain was divided into approximately 6 million mesh cells. The time step of transient simulation was $\sim 4 \cdot 10^{-4} \mathrm{~ns}$.

For the optimal value of magnetic compression $B_{0} / B_{\mathrm{c}}=17.01$, the average radius of the HEB in the central plane of the resonator is $8.5 \mathrm{~mm}$. In the area of excitation of the operating mode $\mathrm{TE}_{12,3}$, the maximum output power was observed at the induction of the 


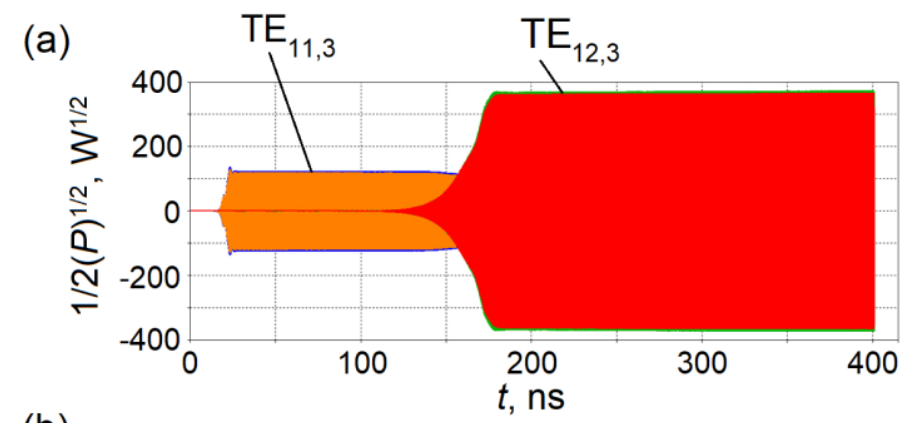

(b)

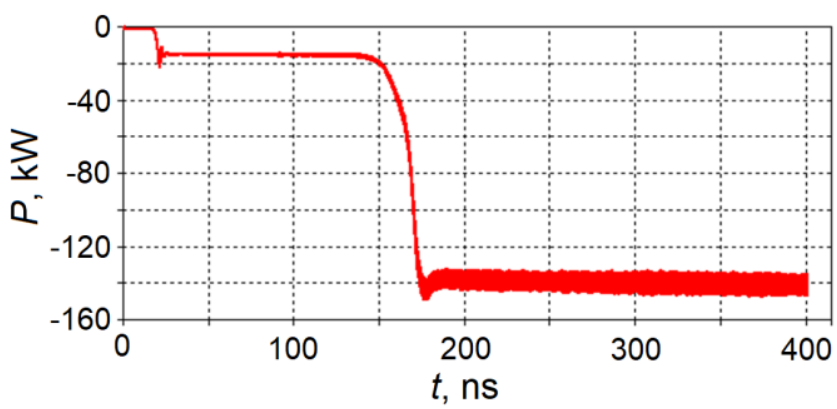

Fig. 2. Time dependence of output port signal in various modes excited in the resonator (a) and waveparticle power transfer (b).

magnetic field in the resonator $B_{0}=2.747 \mathrm{~T}$. Fig. 2, $a$ shows the time variation of the mode signals with highest amplitude in the output port, which were calculated for the uniform cathode at $B_{0} / B_{\mathrm{c}}=17.01$ and $B_{0}=2,747 \mathrm{~T}$. At the beginning of the simulation, the parasitic mode $\mathrm{TE}_{11,3}$ is excited at a frequency of about $\sim 71.4 \mathrm{GHz}$. Afterwards, this mode is suppressed simultaneously with excitation of the working mode $\mathrm{TE}_{12,3}$. During the time period of $180<t<400 \mathrm{~ns}$, stable generation with a frequency of $\sim 74.5 \mathrm{GHz}$ is observed.

The wave-particle power transfer graph (Fig. 2,b) shows that the output microwave power $P_{\mathrm{RF}}=141 \mathrm{~kW}$ with electronic efficiency $\eta_{\mathrm{el}}=47 \%\left(U_{0}=30 \mathrm{kV}, I_{\mathrm{b}}=10 \mathrm{~A}\right)$ is achieved for the uniform cathode to the end of the simulation process $(\mathrm{t}=400 \mathrm{~ns})$ For the sectioned cathode, the modes were excited at the same order and stable generation of the operating mode $\mathrm{TE}_{12,3}$ was characterized by the output power $P_{\mathrm{RF}}=138 \mathrm{~kW}$.

The important information for simulation of the collector energy recovery system is the data on the energy distribution of electrons in the spent HEB after its interaction with highfrequency field. These data were obtained with the particle monitor in the cross-section plane $z=320 \mathrm{~mm}$. This monitor collected data on positions and velocities of particles during the time frame $\Delta t=3 \cdot 10^{-3} \mathrm{~ns}$, which gave us $\sim 25 \cdot 10^{3}$ particles recorded and saved in the output file.

Using equation

$$
\eta_{\mathrm{el}}=1-\frac{\int_{0}^{\infty} f(W) W d W}{e U_{0}},
$$

and the known energy spectrum $f(W)$, the electron efficiency $\eta_{\mathrm{el}}$ can be estimated. For the uniform cathode, the electronic efficiency $\eta_{\mathrm{el}}$ is equal to $47.9 \%$ at the total particle energy $e U_{0}=30 \mathrm{keV}$, which is quite close to the efficiency value determined by the output microwave power. 


\section{Trajectory analysis of spent HEB in the collector region}

The third stage of the combined simulation included trajectory analysis of the spent HEB in the collector with a multistage energy recovery system. Using a method of spatial separation of electrons in the crossed azimuthal magnetic and axial electric fields, a 4-stage depressed collector was designed for the SPbPU gyrotron [17, 24]. The collector model is shown in Fig. 3. Helmholtz coils are used for additional confinement of HEB in the separation region. The azimuthal magnetic field is created by a solenoid with toroidal winding. Magnetic system of the gyrotron provides adiabatic distribution of magnetic field in the transition region between the resonator and the collector. The length of the region, where electrons are decelerated, is about $350 \mathrm{~mm}$. In this region, magnetic field is quasihomogeneous with the magnetic induction values of $0.04 \mathrm{~T}$ and $0.08 \mathrm{~T}$ defined at the beam radius for the axial and azimuthal component correspondingly.

Electric field is created by four electrodes (sections) I-IV of conical form with potentials decreasing when moving away from the resonator (Fig. 3). Radial drift in the crossed electric and magnetic fields leads to spatial separation of electrons with different energies and their deposition on the sections. The collector body and the sections are insulated from each other and from the gyrotron body, which allows implementing a simple one-stage recovery scheme in the case of connected collector body and sections. The collector body has two connecting tubes, inside of which wire "wisps" connecting the inner and outer windings of the toroidal solenoid are placed (Fig. 3). The local magnetic field of the "wisps" influence nearby trajectories of electrons and can lead to their reflection back to the resonator. The use of the sectioned cathode allowed to reduce negative effect of the "wisps" resulting in decrease of the reflection coefficient to $1.37 \%$ of the total beam current.

The goal of trajectory analysis was to ensure optimal separation of electrons and to achieve maximum efficiency of energy recovery, in other words, minimum value of power dissipated in the collector sections $P_{\text {diss. }}$. It should be noted that the collector sections are located in the region where magnetic induction is significantly less than the value $B_{0}$ in the resonator. Therefore, the most part of kinetic energy of electrons is contained in their longitudinal motion along magnetic field lines. The minimum electron energy $W_{\min }$ in the spectrum of the spent HEB determines the value of the voltage $U_{\mathrm{I}}$ applied to the first collector section. In the case of $\left|e U_{\mathrm{I}}\right|>W_{\min }$, electrons with low energies can reflect and move back to the resonator. Reflection of the electrons with higher energies can also occur in the region between the sections. These electrons are not intercepted by the sections after reflection, leave the collector region and move in the adiabatically increasing magnetic field

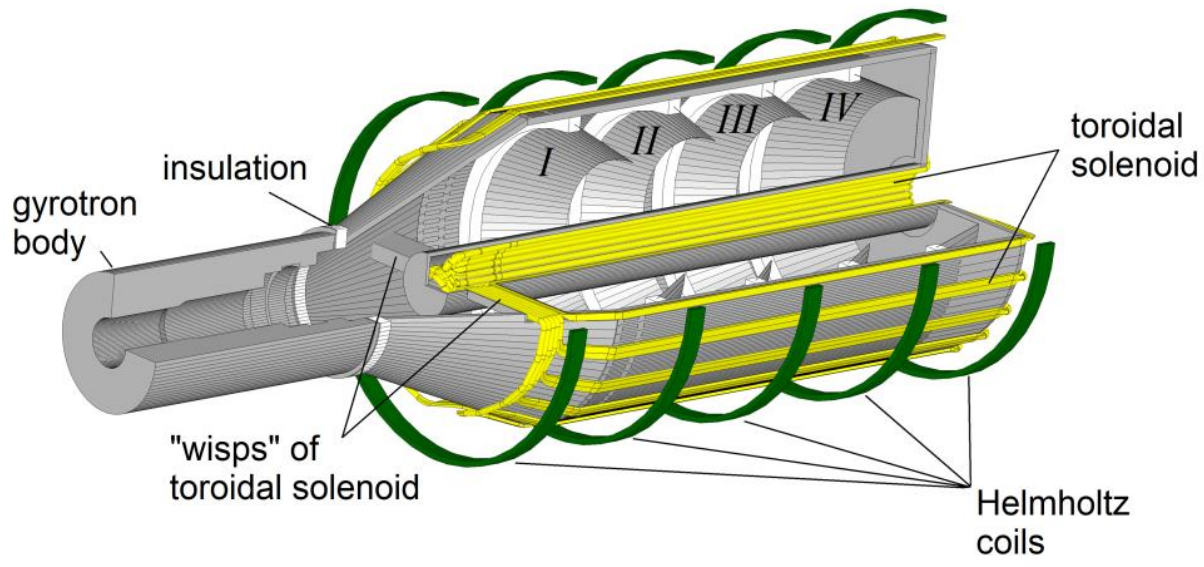

Fig. 3. Schematic drawing of the collector model. 

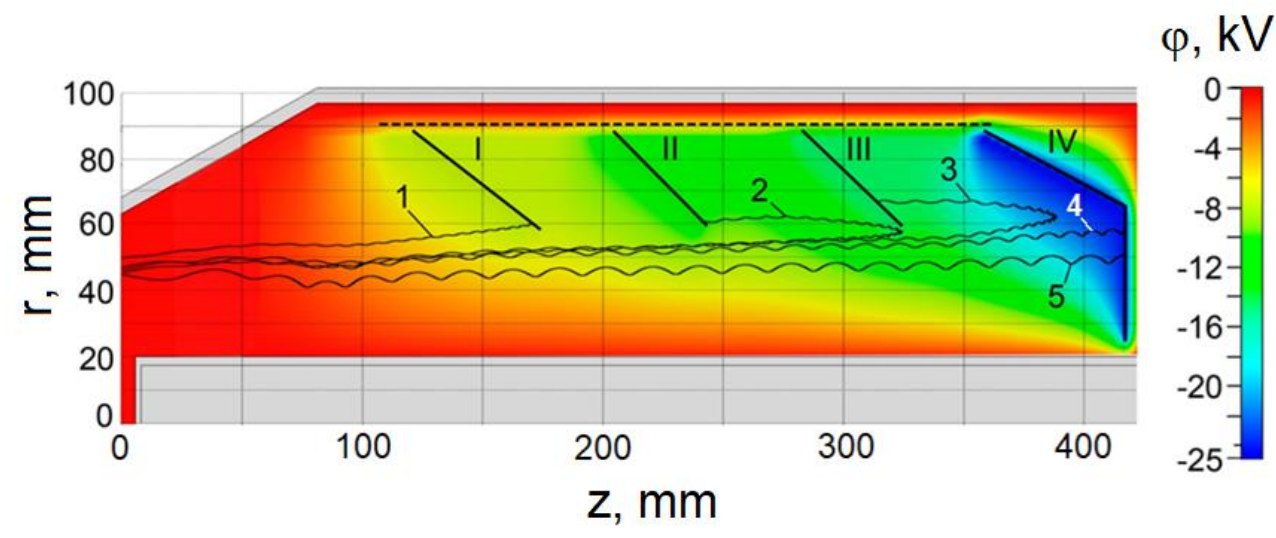

Fig. 4. Electron trajectories 1-5 and distribution of potential $\varphi$ in $r-z$ cross-section. Elements of the magnetic system are hidden.

in the direction to the resonator. Reflected electrons can interact with high-frequency field, which, in principle, negatively affects the values of output power and electronic efficiency. Previous investigations show that the acceptable level of reflection from the collector can differ in different gyrotrons [5, 25-27] and ultimately be limited by $10 \%$ [28].

The complex problem was solved to determine optimal geometry and potentials of the collector sections providing the minimum value of dissipated power $P_{\text {diss }}$ at the electron reflection coefficient $R_{\text {ref }}$ of $1-2 \%$. As a result of the optimization, the following values of the section potentials were determined: $U_{\mathrm{I}}=-7.72 \mathrm{kV}, U_{\text {II }}=-10.72 \mathrm{kV}, U_{\text {III }}=-14.72 \mathrm{kV}$, $U_{\mathrm{IV}}=-24.72 \mathrm{kV}$ at the potential of the collector body equal to 0 . In this regime, the dissipated power $P_{\text {diss }}=54.19 \mathrm{~kW}$ and the reflection coefficient $R_{\text {ref }}=1.37 \%$ were calculated.

Projections of electron trajectories in the $r-z$ plane of the collector region and electric potential distribution are illustrated in Fig. 4. Electrons with different energy, pitch ratio and initial radial coordinate in the input plane $z=320 \mathrm{~mm}$ were selected (Table 2). These electrons pass through the transition region at approximately equal distance from the "wisps". The average pitch ratio $\bar{\alpha}$ in the spent HEB $(z=320 \mathrm{~mm})$ is $\sim 0.59$. With increasing energy of particles, their pitch ratio will also increase (Table 2), that is a consequence of the mechanism of exchange of energy between HEB and high-frequency field in the resonator. The presented trajectories demonstrate correct operation of the method of spatial separation of electrons in crossed $E_{\mathrm{z}} \times B_{\theta}$ fields, which provides deposition of particles with different energies on the sections under corresponding potentials.

Considering the calculated dissipated power $P_{\text {diss }}$, total efficiency of the gyrotron can be calculated from the following:

Table 2. Input parameters of electron trajectories.

\begin{tabular}{|c|l|l|l|}
\hline № & Energy, keV & Pitch ratio & Radius, mm \\
\hline 1 & 8.270 & 0.45 & 9.34 \\
\hline 2 & 13.781 & 0.45 & 8.7 \\
\hline 3 & 21.264 & 0.74 & 9.04 \\
\hline 4 & 28.097 & 0.83 & 9.03 \\
\hline 5 & 46.368 & 1.65 & 8.63 \\
\hline
\end{tabular}




$$
\eta_{\mathrm{t}}=\frac{P_{\mathrm{RF}}}{P_{\mathrm{RF}}+P_{\text {diss }}}=71.8 \%,
$$

where $P_{\mathrm{RF}}=138 \mathrm{~kW}, P_{\text {diss }}=54.19 \mathrm{~kW}$. The energy recovery efficiency of the collector is $66.5 \%$. In this system, the main part of electrons was deposited on the first section, which was caused by the necessity to reduce the inner radius of the first section and to reduce $\left|e U_{\mathrm{I}}\right|$ in order to provide relatively low reflection of electrons. The main sources of reflected electrons were disturbing influence of the local magnetic field of the "wisps" and the spread of initial radial coordinates of electrons. If the acceptable level of reflection is increased, it is possible to achieve a further increase in total efficiency. Note, that if we consider a 4stage depressed collector with ideal separation when each electron is deposited on a section with a potential corresponding to its energy, the maximum total efficiency is $81.2 \%$ for the residual energy of electrons $W_{\text {res }}>0.5 \mathrm{keV}$ and $83.6 \%$ for $W_{\text {res }}>0$. These estimations were made for the spectrum of the spent HEB obtained for the sectioned cathode assuming that the electron reflection coefficient is equal to $1.37 \%$.

The possibilities for further increasing the efficiency of the developed method of electron energy recovery are obviously associated with improving the design of the magnetic system that provides the required distribution of the azimuthal magnetic field. The authors continue to search for optimal method to create such a field, that would increase the total efficiency of gyrotrons including powerful gyrotrons operating in long-pulse and quasi-continuous regimes.

\section{Conclusion}

Within the framework of this study, the combined simulation of a moderate-power pulsed 4-mm gyrotron was performed. It is shown that record values of gyrotron total efficiency can be achieved as a result of improving the quality of HEB in the gyrotron electron-optical system and implementing a system of multistage recovery of residual energy of electrons. A 4-stage depressed collector system based on applying crossed azimuthal magnetic and axial electric fields was designed. Using a sectioned cathode with two azimuthal sectors with suppressed emission, the negative effect of the "wisps" of the toroidal solenoid can be reduced and the coefficient of electron reflection from the collector can be decreased. The total gyrotron efficiency of $71.8 \%$ was achieved at the reflected current of $1.37 \%$ of the HEB total current.

The study was performed by a grant of the Russian Science Foundation (project No. 16-12-10010). Part of the results were obtained using the computing resources of the Supercomputer Center of the Peter the Great St. Petersburg Polytechnic University (http://www.scc.spbstu.ru).

\section{References}

1. A.G. Litvak, G.G. Denisov, V.E. Myasnikov, et al., J. Infrared Millim. Terahertz Waves 32, 337 (2011)

2. M. Thumm State-of-the-art of high power gyro-devices and free electron masers, update 2017, KIT Scientific Report 7750, 183 (2018)

3. V.E. Zapevalov, Radiophys. Quantum Electron. 49, 779 (2006)

4. Sh.E. Tsimring, Int. J. Infrared Millim. Waves 22, 1433 (2001)

5. N.A. Zavolskiy, V.E. Zapevalov, A.N. Kuftin and A.S. Postnikova, Proceedings of CriMiCo'2018, pp. 1131-1137 (2018)

6. O.I. Louksha, Helical electron beams of gyrotrons: dynamics of space charge and methods of quality increase (SPbPU, Saint Petersburg, 2011) 
7. D.V. Kas'yanenko, O.I. Louksha, B. Piosczcyk, ,Low-frequency parasitic spacecharge oscillations in the helical electron beam of a gyrotron, Radiophys. Quantum Electron. 47, pp. 414-420 (2004)

8. O.I. Louksha, B. Piosczyk, G.G. Sominski, M.K. Thumm and D.B. Samsonov, On potentials of gyrotron efficiency enhancement: measurements and simulations on a 4mm gyrotron, IEEE Trans. Plasma Sci. 34, pp. 502-511 (2006)

9. O.I. Louksha, D.B. Samsonov, G.G. Sominski and A.A. Tsapov, Improvement of electron beam quality and the gyrotron efficiency by controlling the electric field distribution near a magnetron injection gun, Technical phys. 57, pp. 835-839 (2012)

10. O.I. Louksha, D.B. Samsonov, G.G. Sominski and S.V. Semin, Dynamic processes in helical electron beams in gyrotrons, Technical Phys. 58, pp. 751-759 (2013)

11. O.I. Louksha, G.G. Sominski, A.V. Arkhipov, et al., Gyrotron research at SPbPU: diagnostics and quality improvement of electron beam, IEEE Trans. Plasma Sci. 44, 1310 (2016)

12. V.N. Manuilov, M.V. Morozkin, O.I. Luksha and M.Y. Glyavin, Gyrotron collector systems: types and capabilities, Infrared Physics and Technology 91, pp. $46-54$ (2018)

13. H.G. Kosmahl, Proceedings of IEEE Conf. 70, pp. 1325-1334 (1982)

14. I.Gr. Pagonakis, J.-P. Hogge, S. Alberti, K.A. Avramidis and J.L. Vomvoridis, IEEE Trans. Plasma Sci. 36, pp. 469-480 (2008)

15. O.I. Louksha and P.A. Trofimov, A method of electron separation for multistep recuperation systems in gyrotrons, Technical Phys. Lett. 41, pp. 884-886 (2015)

16. C. Wu, I.G. Pagonakis, K.A. Avramidis, et al., Phys. of Plasmas 25, 033108 (2018)

17. O.I. Louksha and P.A. Trofimov, A multistage depressed collector with azimuthal magnetic field for gyrotrons, Proceedings of IRMMW-THz'2016, 7758519 (2016)

18. http://www.cst.com

19. O.I. Louksha and P.A. Trofimov, Simulation of non-uniform electron beams in the gyrotron electron-optical system, Technical phys. 63, pp. 598-604 (2018)

20. E.G. Avdoshin, L.V. Nikolaev, I.N. Platonov and Sh.E.Tsimring, Radiophys. Quantum Electron. 16, pp. 461-466 (1973)

21. J. Zhang, S. Illy, I.Gr. Pagonakis, et al., Nuclear Fusion 56, 026002 (2015)

22. V.N. Manuilov, Radiophys. Quantum Electron. 49, pp. 786-792 (2006)

23. O.I. Luksha, Simulation of low-frequency collective processes in gyrotron electron beams, Radiophys. Quantum Electron. 52, 386 (2009)

24. O.I. Louksha and P.A. Trofimov, A multistage depressed collector system for gyrotrons, Proceedings of the $18^{\text {th }}$ International Vacuum Electronic Conference, pp. $1-2(2017)$

25. K. Sakamoto, M. Tsuneoka, A. Kasugai, et al., Phys. Rev. Lett. 73, pp. 3532-3535 (1994)

26. N.P. Venediktov, M.Yu. Glyavin, V.E. Zapevalov and A.N.Kuftin, Radiophys. Quantum Electron. 41, pp. 449-456 (1998)

27. M.V. Morozkin, M.Y. Glyavin, G.G. Denisov and A.G. Luchinin, Int. J. Infrared Millimeter Waves 29, pp. 1004-1010 (2008)

28. B. Piosczyk, C.T. Iatrou, G. Dammertz and M. Thumm, IEEE Trans. Plasma Sci. 24, pp. 579-585 (1996) 